\title{
Extracts of Different Organs of Macadamia Nut Tree (Macadamia Integrifolia) Ameliorate Oxidative Damage in D-Galactose Accelerated Aging Model in Rats
}

\author{
Seham El-Hawary 1(i), Mohammed Abubaker ${ }^{2,3}$ (D), Engy A. Mahrous ${ }^{1, *(\mathbb{C})}$ \\ 1 Pharmacognosy Department, Faculty of Pharmacy, Cairo University, Cairo, Egypt, seham.elhawary@pharma.cu.edu.eg \\ (S.S.); engy.abdelhamid@pharma.cu.edu.eg (E.A.M.); \\ 2 Pharmacy department, Thamar University, Dhamar, Republic of Yemen \\ 3 Department of Medicine, McGill University Health Center Research Institute, McGill University, Montreal, Quebec, \\ Canada, banabila550@gmail.com (M.A.); \\ * Correspondence: engy.abdelhamid@pharma.cu.edu.eg (E.A.M);
}

Scopus Author ID 13006535500

Received: 28.09.2021; Revised: 1.11.2021; Accepted: 5.11.2021; Published: 21.11.2021

\begin{abstract}
Macadamia nut tree, Macadamia integrifolia (Maiden \& Betche), is cultivated for the production of the edible macadamia nuts, which are a good source of monounsaturated fatty acids. We investigated the effect of ethanolic extracts of leaves, nuts, and nutshells of macadamia in D-galactose accelerated aging model in rats. Administration of D-galactose $(150 \mathrm{mg} / \mathrm{kg})$ in rats for 60 days resulted in impairment of cognitive function and motor coordination and caused an increase in oxidative stress and deterioration of liver and kidney functions. Macadamia nut extract ameliorated cognitive impairment induced by D-galactose as inferred from Morris water maze test and balance test using rotarod. Also, nut extract was superior to leaves and shell extract in reducing serum levels of malondialdehyde $(50 \%)$, alanine transaminase $(63 \%)$, aspartate transaminase $(63 \%)$, total bilirubin $(24 \%)$, creatinine $(38 \%)$, and urea (16\%) compared to animals that received no treatment. Chemical analysis showed that macadamia nut extract has a high percentage of oleic acid (81\%) followed by palmitoleic acid (6.9\%). This study encourages further investigation of the health benefits of macadamia nuts and the underlying mechanism of these effects.
\end{abstract}

Keywords: Macadamia nut; D-galactose aging model; cognitive impairment; oleic acid; palmitoleic acid.

(C) 2021 by the authors. This article is an open-access article distributed under the terms and conditions of the Creative Commons Attribution (CC BY) license (https://creativecommons.org/licenses/by/4.0/).

\section{Introduction}

Macadamia nut is the fruit kernel obtained from two species of the genus Macadamia: M. integrifolia (Maiden \&Betche) and M. tetraphylla (L.A.S Johnson), family Proteaceae. Macadamia integrifolia, the smooth-shelled macadamia, is indigenous to the subtropical coastal region of Australia but is currently cultivated in different areas around the world, including Hawaii and South Africa [1,2]. Macadamia nuts are a rich source of monounsaturated fatty acids MUFA [3,4] as well as plant sterols where $\beta$-sitosterol, 5-avenasterol, campesterol, stigmasterol were identified as major constituents [5]. Meanwhile, macadamia nuts have relatively low phenolic content compared to other edible nuts such as pistachios [5], and its major phenolic constituent is reported to be the cyanogenic glycoside dhurrin $[6,7]$. It should be noted that apart from the edible nuts, other plant organs have not been fully investigated for their phytochemical constituents $[3,8]$ 
Macadamia nut oil is often incorporated in skin creams, especially those intended as anti-aging preparations due to their high content of MUFA and Vitamin E [9,10]. Its high content of MUFA may also be responsible for the decreased risk of coronary artery diseases and improved blood lipid profile observed in hypercholesterolemic human subjects who incorporated macadamia nuts in their diet [11-13]. Additionally, preliminary animal experiments found that supplementation with macadamia oil can affect anthropometric indices in obese individuals [14].

In this study, we investigate the effect of extracts obtained from different plant organs in deterring signs of senescence induced by the administration of D-galactose. Accelerated aging by D-galactose in rats is a widely used model for discovering anti-aging therapeutics $[15,16]$. D-galactose is converted in the body to aldose at high doses, releasing reactive oxygen species and disrupting oxidative balance. The subsequent oxidative stress causes significant inflammation and loss of proper cellular function, negatively affecting vital organs, immune response, and neurological functions, ultimately reducing life expectancy [17]. Herein we study the effect of administration of macadamia nut extract (MNE) and extracts obtained from agricultural waste, including macadamia leaves extract (MLE) and macadamia shell extract (MSE) in D-galactose aged rats with particular focus on their effects on the liver, kidney, and neurological functions.

\section{Materials and Methods}

\subsection{Plant material.}

Leaves and seeds of Macadamia integrifolia Maiden \& Betche used in this study were collected in March 2017 from Horticulture Research Institute, Agriculture Research Center, Giza, Egypt. Dr. Reem Sameer Hamdi, assistant professor of plant taxonomy at Department of Botany, Faculty of Sciences, Cairo University, kindly authenticated plant identity. A voucher specimen (19.9.16) was deposited at the herbarium of Pharmacognosy Department, Faculty of Pharmacy, Cairo University

\subsection{Chemicals.}

Dhurrin, protocatechuic acid, and gallic acid were isolated from leaves extract as described previously [18]. $\alpha$-Tocopherol, kaempferol, and hesperidin were kindly supplied by Food Technology Research Institute, Giza, Egypt. D-galactose was purchased from SigmaAldrich (St'Louis, MO, US). Different fatty acids methyl esters used as external standards for gas chromatography analysis were supplied by National Research Center, Giza, Egypt.

\subsection{Preparation of plant extract.}

$200 \mathrm{~g}$ of each of the air-dried powder kernels, shells, and leaves of M. integrifolia were separately macerated in successive portions of ethyl alcohol $95 \%$ till exhaustion. The alcoholic extracts were pooled and evaporated under vacuum to obtain 77.14, 36.8, and $41 \mathrm{~g}$ residues of MNE, MSE, and MLE, respectively.

\subsection{Gas chromatography analysis of the fatty acid composition of extracts.}

Two grams of each extract were saponified with $10 \%$ ethanolic $\mathrm{KOH}(50 \mathrm{~mL})$ under reflux conditions [19]. After shaking with ether to recover unsaponifiable matter, the aqueous 
solution was acidified and extracted repeatedly with ether. The combined ether extract was methylated using a concentrated $\mathrm{HCl}$ : methanol: water mixture described previously to produce fatty acid methyl esters, FAMEs [20]. Ether was used to recover FAMEs, which were injected into a gas chromatography system (Agilent 6890) equipped with capillary column: TG-5MS fused silica ( $30 \mathrm{~m} \times 0.32 \mathrm{~mm}$, film thickness $0.25 \mu \mathrm{m}$ ). Helium was used as a carrier gas, and the temperature program started at $50{ }^{\circ} \mathrm{C}$ for 3 minutes, then to $300^{\circ} \mathrm{C}$ at a rate $5^{\circ} \mathrm{C} / \mathrm{min}$, then isothermal at $300{ }^{\circ} \mathrm{C}$ for 5 minutes. Eluted peaks were identified based on their relative retention time in comparison to FAMEs standards.

\subsection{HPLC analysis of extracts.}

HPLC analysis was performed using Hewlett Packard (Series 1050) equipped with solvent degasser, ultraviolet (UV) detector set at $280 \mathrm{~nm}$, and Phenomenex C18 column (250 $\mathrm{mm} \times 4.6 \mathrm{~mm}$ ) maintained at a temperature at $35^{\circ} \mathrm{C}$. Separation was achieved isocratic using methanol:acetic acid: water (36:0.9:63.1, v/v/v) as a mobile phase at a flow rate of $1 \mathrm{~mL} / \mathrm{min}$. External standards were dissolved in the mobile phase at concentration $0.1-10 \mathrm{mg} / \mathrm{mL}$ and analyzed under the same conditions to establish a standard calibration curve. $\alpha$-tocopherol content was determined according to the method described by Pyka \& Sliwiok [21].

\subsection{Effect of extract in D-galactose accelerated aging animal model.}

\subsubsection{Animals.}

Forty Wister albino male rats weighing from 200-225 g were used for D-galactose induced toxicity experiment. The animals were obtained from the animal house colony of the National Research Centre, Giza, Egypt, and were housed in standard metal cages in an airconditioned room at $22 \pm 3{ }^{\circ} \mathrm{C}$ and $55 \pm 5 \%$ humidity. Animals were provided with a standard laboratory diet and water ad libitum. All experimental procedures were conducted in accordance with the guide for the care and use of laboratory animals and in accordance with the Ethics Committee of the National Research Centre and followed the recommendations of the National Institutes of Health guides for care and use of laboratory animals.

\subsubsection{D-galactose accelerated aging model.}

Animals were divided into five groups (each containing 8 rats). Group 1 served as normal control receiving saline, and the rest were treated with D-galactose in dose $150 \mathrm{mg} / \mathrm{kg}$ i.p once a day for 60 days using saline as a vehicle[22]. After 60 days, group 2 received saline only for 30 days while groups 3, 4, 5 received intragastrically daily doses ( $200 \mathrm{mg} / \mathrm{kg} / \mathrm{day})$ of the investigated extracts MNE, MSE, and MLE, respectively for 30 days.

\subsubsection{Memory assessment using Morris's water maze.}

The water maze contained a circular water pool designed as described originally by Morris [23]. Throughout the four days, acquisition trials, an escape platform was maintained in a constant location in the northwest NW quadrant. Rats were trained to locate this hidden platform, and animals that failed to find the platform within $20 \mathrm{sec}$ by the 4th trial day were excluded from the study. On probe day (day5), $24 \mathrm{~h}$ after the last acquisition trial, the escape platform was removed, and a retention trial was conducted. The animals were allowed to swim for $60 \mathrm{~s}$ before the end of the session. Retention trials were repeated on day 90 on all groups to 
evaluate memory consolidation. Data were obtained through a video camera attached to a computerized tracking system fixed above the center of the pool. Time to reach the hidden platform (escape latency), latency to find the target quadrant (NW latency), and percentage of time spent in the target quadrant (NW) were measured.

\subsubsection{Motor coordination assessment.}

Motor coordination and balance were assessed using a rotarod apparatus (Med Associates, Italy) [24]. Four animals at a time were placed on a rod rotating at $30 \mathrm{rpm}$ speed. Only the rats that demonstrated their ability to remain on the revolving rod for $5 \mathrm{~min}$ after training sessions were selected for studies. The fall-off time was recorded in all the groups. Motor coordination was assessed on the 83rd day before the probe trial in the Morris water maze.

\subsubsection{Biochemical evaluation.}

Animals were mildly anesthetized at the end of the experimental period with fluothane, and blood samples were collected by retroorbital sinus puncture into micro-centrifuge tubes. Blood was allowed to clot for half an hour and then centrifuged at $10000 \mathrm{rpm}$ for 10 minutes to obtain serum. Collected sera were divided into aliquots and stored at $-20{ }^{\circ} \mathrm{C}$. Serum concentration of alanine transaminase (ALT) and aspartate transamiase (AST) were estimated using kits by Quimica Clinica (Aplicada, Spain). Bilirubin, urea, and creatinine concentrations were measured using commercial kits by Bio-diagnostics, Egypt. Oxidative stress markers, i.e., reduced glutathione (GSH) and malondialdehyde (MDA) were estimated as described previously $[25,26]$.

\subsubsection{Histopathological examination.}

Liver specimens were fixed in $10 \%$ formaldehyde and subsequently embedded in paraffin and sliced into slices of $4 \mu \mathrm{m}$ thickness followed by staining with hematoxylin and eosin (H\&E) and examined under a light microscope.

\subsection{Statistical analysis.}

Data were analyzed by one-way analysis of variance (ANOVA) followed by Tukey's post hoc test. Data were expressed as mean \pm standard error, and the values of $\mathrm{P}<0.05$ were considered statistically significant.

\section{Results and Discussion}

\subsection{Chemical characterization of extracts.}

Although not widely investigated, the macadamia nut is known for its high-fat content (around 70\% w/w) [4,27] and is recommended as a healthy diet for its high content of MUFA, at $65-83 \%$ [28]. Additionally, the edible kernel is one of the richest natural sources of palmitoleic acid [29]. $\beta$-Sitosterol, 5-avenasterol, catechin, epicatechin, $\alpha$-tocopherol, gallic acid, galactolipids, and dhurrin are among the few secondary metabolites identified in $M$. integrifolia [7,18]. Our investigation confirmed the previously reported data as monounsaturated fatty acids, MUFA, including oleic acid and palmitoleic acids, were the major fatty acids identified in macadamia nuts and shells for a total MUFA content at $88.26 \%$ and 
$69.4 \%$, respectively, Table 1. Meanwhile, leaves extract, which has not been investigated for its fatty acid content, showed a high percentage of saturated fatty acids and lowered MUFA content at 32.7\%. Compared to MNE and MSE, leaves extract was more enriched in secondary metabolites, including the highest $\alpha$-tocopherol content at $8.87 \mathrm{mg} / \mathrm{g}$, Table 2 .

Table 1. Fatty acid methyl esters (FAMEs) identified in macadamia extract and represented as a percentage of the total FAME.

\begin{tabular}{l|c|c|c|c|c|c}
\multicolumn{2}{c}{ Identified fatty acids } & Rt & RRt & MLE & MNE & MSE \\
\hline \multirow{4}{*}{ Saturated fatty acids } & Myristic acid & 33.6 & 0.78 & 2.78 & 0.1 & 0.65 \\
\cline { 2 - 7 } & Palmitic acid & 38.71 & 0.9 & 42.13 & 5.81 & 15.77 \\
\cline { 2 - 7 } & Margaric acid & 41 & 0.95 & 1.44 & -- & 0.33 \\
\cline { 2 - 7 } & Stearic acid & 43.66 & 1.01 & -- & 2.35 & 2.51 \\
\cline { 2 - 7 } & Arachidic acid & 48.29 & 1.12 & -- & 1.02 & 1.01 \\
\cline { 2 - 7 } & Behenic acid & 52.62 & 1.22 & -- & 0.19 & 0.68 \\
\cline { 2 - 7 } & Lignoceric acid & 56.6 & 1.31 & -- & 0.06 & 1.09 \\
\hline \multirow{3}{*}{ Unsaturated fatty acid } & Palmitoleic acid & 38.18 & 0.89 & 0.96 & 6.93 & 3.29 \\
\cline { 2 - 7 } & Oleic acid & 43.1 & 1 & 31.77 & 81.33 & 66.13 \\
\cline { 2 - 7 } & Linoleic acid & 42.9 & 0.99 & 14.7 & 0.21 & 5.59 \\
\hline Percentage of identified components & & & 43.78 & 98 & 97.05 \\
\hline Percentage of saturated fatty acids & & & 47.43 & 9.53 & 22.04 \\
\hline Percentage of unsaturated fatty acid
\end{tabular}

MLE: macadamia leaves extract, MNE: macadamia nuts extract, MSE: macadamia shells extract, Rt: retention time, RRt: retention time relative to oleic acid

Similarly, HPLC analysis of all three extracts showed the highest concentrations of dhurrin, gallic acid, and protocatechuic acid were observed in leaves extract, MLE, while extract of the edible nut kernel (MNE) had a relatively low content of these phenolic compounds, Table 2 .

Table 2. Concentration of major phenolic compounds identified by HPLC and expressed as mg per gram of

\begin{tabular}{l|l|l|l|l} 
Compound & Rt(min) & MLE & MNE & MSE \\
\hline Gallic acid & 3.56 & $2.5 \pm 0.09$ & $0.75 \pm 0.03$ & $2.4 \pm 0.01$ \\
\hline Dhurrin & 3.92 & $124.2 \pm 0.6$ & $21.07 \pm 0.2$ & $74.73 \pm 0.7$ \\
\hline $\begin{array}{l}\text { Protocatechuic } \\
\text { acid }\end{array}$ & 4.503 & $9.3 \pm 0.03$ & $1.36 \pm 0.02$ & $3.04 \pm 0.03$ \\
\hline Hespiridin & 6.29 & 0.05 & 0.001 & 0.001 \\
\hline Kaempferol & 10.27 & 0.04 & 0.004 & - \\
& \multicolumn{5}{c}{ Data are mean \pm SD $(\mathrm{n}=3)}$. &
\end{tabular}

\subsection{Effect of extracts on brain functions.}

We investigated the effect of different macadamia extract in D-galactose accelerated aging animal models. This model is widely used to study aging both in cell culture and in experimental animals receiving high galactose doses over a period of 6-8 weeks[17,30]. The model has been validated through several studies that reported an increase in biomarkers of aging such amyloid $\beta$-protein, advanced glycation end products (AGE), shortening of telomere length, and increased activity of telomerase [17]. Rats treated with D-galactose for 60 days showed significant impairment in memory as indicated by the Morris water maze test, Table 3. During the probe trial on day 90, D-galactose-treated animals that received no other treatment spent significantly less time in the target quadrant $(\mathrm{NW})$ than the control group. This memory deficit caused by $\mathrm{D}$-galactose was significantly reversed $(P<0.05)$ by all investigated extracts, where MNE treatment (200 mg/kg/day) reduced escape latency time (ELT) by $63 \%$ and increased the time spent at the target quadrant (NW) to $81.5 \%$ compared to normal animals at $85 \%$ (Table 3). MLE and MSE were slightly less effective, causing reduction of ELT by $61 \%$ 
and $57 \%$, respectively, when compared to D-galactose treated animals while increasing percentage of time at NW quadrant to 78 and $75 \%$, respectively, Table 3.

The effect of D-galactose on balance and coordination was tested on an accelerating rotarod. All animals in D-galactose treated group showed more propensity to fall than the normal group with reduced latency time (Table 3). Administration of MNE prolonged latency to fall to $95 \%$ of the value observed in normal untreated animals (control group), while administration of MSE and MLE restored latency time to fall to 89.4 and $84.2 \%$ of the value reported for the control group, respectively (Table 3).

Table 3. Effect of tested extracts on cognitive impairment and motor coordination caused by D-galactose.

\begin{tabular}{l|c|c|c|c} 
Groups & ELT (S) & NW latency & $\begin{array}{l}\text { \% of the time } \\
\text { in target quad }\end{array}$ & $\begin{array}{l}\text { Latency to } \\
\text { fall (s) }\end{array}$ \\
\hline Normal & $18.5 \pm 0.23$ & $51 \pm 1.3$ & 85 & $285 \pm 3$ \\
\hline D- galactose & $57.7 \pm 1.2^{\alpha}$ & $27 \pm 1.2^{\alpha}$ & 45 & $187 \pm 12^{\alpha}$ \\
\hline Leaves & $24.6 \pm 1.7^{*}$ & $45 \pm 1.7^{*}$ & 75 & $240 \pm 7^{*}$ \\
\hline Pericarp & $22.5 \pm 0.9^{*}$ & $47 \pm 1.9^{*}$ & 78 & $255 \pm 9^{*}$ \\
\hline Kernel & $21.3 \pm 5^{*}$ & $49 \pm 2.5^{*}$ & 81.5 & $271 \pm 5^{*}$
\end{tabular}

Data are mean $\pm \mathrm{SD}(\mathrm{n}=8) .{ }^{\alpha}$ Significant versus control $(\mathrm{P}<0.05),{ }^{*}$ Significant versus $\mathrm{D}$-galactose $(\mathrm{P}<0.05)$.

\subsection{Effect on serum oxidative stress biomarkers.}

Animals that received only vehicle after treatment with D-galactose for 60 days showed marked elevation in serum levels of MDA by 2.2 fold and a decrease in serum GSH by $52 \%$ $(\mathrm{P}<0.05)$ of the normal control value, Table 4. Administration of $\mathrm{MNE}$ at $200 \mathrm{mg} / \mathrm{kg}$ daily to rats treated with D-galactose caused a $47.3 \%$ increase in serum GSH and a 50\% decrease in MDA compared to animals treated with vehicle only. Administration of MNE seemed to totally reverse oxidative stress status as it restored GSH to more than $97 \%$ of its levels in normal animals and resulted in less than 10\% increase in MDA compared to the normal control group. Among the three tested extracts, MLE was the least effective in eliminating oxidative stress restoring GSH to $80 \%$ of its basal level and maintaining a $28.6 \%$ increase in MDA compared to normal control, Table 4.

Table 4. Effect of tested extracts on oxidative stress parameters in D-galactose accelerated aging animals.

\begin{tabular}{l|c|c} 
Groups & GSH $(\mu \mathbf{M} / \mathbf{m L})$ & $\begin{array}{c}\text { MDA } \\
(\mathbf{m m o l} / \mathbf{m L})\end{array}$ \\
\hline Normal & $7.3 \pm 0.91$ & $8.4 \pm 0.39$ \\
\hline D- galactose & $3.53 \pm 0.41 \alpha$ & $18.5 \pm 1.23 \alpha$ \\
\hline MLE & $6.5 \pm 0.44^{*}$ & $10.6 \pm 0.84^{*}$ \\
\hline MSE & $6.7 \pm 0.35^{*}$ & $9.9 \pm 0.57^{*}$ \\
\hline MNE & $7.1 \pm 0.28^{*}$ & $9.2 \pm 0.47^{*}$
\end{tabular}

Data are mean $\pm \mathrm{SD}(\mathrm{n}=8) .{ }^{\alpha}$ Significant versus control $(\mathrm{P}<0.05),{ }^{*}$ Significant versus $\mathrm{D}$-galactose $(\mathrm{P}<0.05)$.

\subsection{Effect of extracts on liver functions.}

The serum concentration of alanine transaminase (ALT), aspartate transaminase (AST), and total bilirubin were elevated by 3.1, 3.6, and 1.4 folds, respectively, in animals receiving D-galactose, only indicating deterioration of liver functions. Administration of MNE significantly reduced serum concentration of ALT, AST, and bilirubin by 63.4, 63.3, and 23.9\%, respectively, as shown in Figure 1. Administration of MSE and MLE caused a significant reduction in the elevated levels of these biomarkers $(\mathrm{P}<0.05)$, albeit to less extent with MLE causing the least effective among the three investigated extracts causing a reduction in serum ALT, AST, and total bilirubin by 55.3, 47.3 and 16.9\%, respectively, Figure 1. 
The detrimental effect of D-galactose on the liver was further assessed by histopathological examination. Liver sections from rats receiving D-galactose for 60 days showed many pathological features, including focal hepatic necrosis associated with inflammatory cells infiltration and vacuolated nuclei and cystic dilation of the bile duct and fibroplasia in the portal triad, Figure 2 C, D. Administration of MNE at $200 \mathrm{mg} / \mathrm{g}$ greatly improved the alterations in liver morphology to the extent that no pathological alteration was observed, Figure 2 E, F. Administration of MSE caused only mild histopathological changes where congestion of central vein was observed, Figure $2 \mathrm{G}, \mathrm{H}$. Meanwhile, several histopathological changes were observed in the hepatic tissue of animals treated with 200 $\mathrm{mg} / \mathrm{kg}$ of MLE, Figure $2 \mathrm{I}$, J.

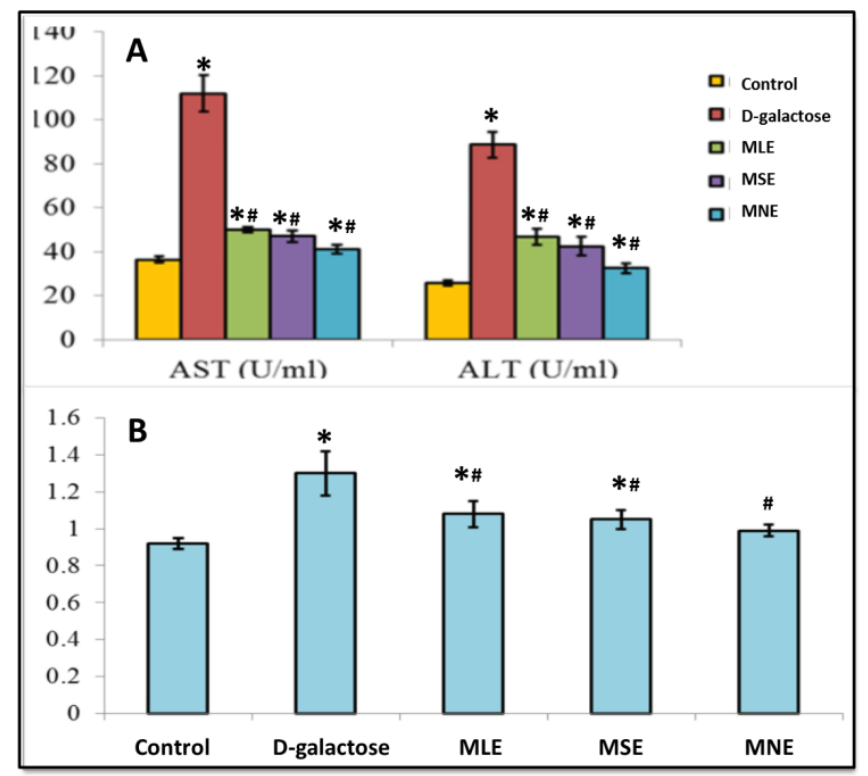

Figure 1. Effect of tested extracts on liver function biomarkers in D-galactose accelerated aging animals. Serum activity of AST and ALT as U/mL (A) and total bilirubin concentration in $\mathrm{mg} / \mathrm{dL}$ (B) measured in different animal groups. Except for the control group, all animals were treated with D-galactose for 60 days

$(150 \mathrm{mg} / \mathrm{kg}$ ) and received no further treatment (D-galactose ) or received $200 \mathrm{mg} / \mathrm{Kg} /$ day for 30 days of macadamia leaves extract (MLE), macadamia shells extract (MSE) and macadamia nut extracts (MNE).

* significantly different from control animals $(\mathrm{P}<0.05)$, \# significantly different from $\mathrm{D}$-galactose treated animals $(\mathrm{P}<0.05)$.

\subsection{Effect of extracts on kidney functions.}

Similar to liver functions, biomarkers indicators for kidney functions were elevated in D-galactose-treated rats. Accelerated aging by D-galactose resulted in a 1.8 and 1.3 fold increase in serum concentration of creatinine and urea while causing a reduction in serum proteins by $35 \%$, Figure 3. Administration of any of the three investigated extracts caused a significant reduction of creatinine (19.4-37.8\%) and elevation in total serum protein (35.3$60.7 \%$ ), $\mathrm{P}<0.05$. Meanwhile, the effect of extracts in serum urea level was less prominent (4$16 \%$ ), Figure 3. It is worth mentioning that MNE showed better overall restoration of kidney functions among the three investigated extracts.

In order to correlate the observed activity to the chemical composition of each extract, it is important to note that macadamia nuts are far richer in fats and proteins $(68 \%, 20.8 \mathrm{w} / \mathrm{w})$ when compared to leaves (5 and 6.6\%, respectively), [27]. Interestingly, while leaves extract was more enriched in phenolic antioxidant compounds such as $\alpha$-tocopherol, gallic, protocatechuic acids, and dhurrin than shells and nut extract, it showed the least activity in our 
in vivo model, including the least ability to reverse oxidative stress. Therefore, it can be suggested that a high percentage of oleic acid and MUFA in MNE and MSE may play a more significant role in restoring brain functions and oxidative balance than phenolic compounds. In support of this theory, oleic acid has been shown to exert a neuroprotective effect mediated through PPAR- $\gamma$ anti-inflammatory action [31]. Furthermore, other vegetable oils enriched in oleic acid (such as olive oil) are known to have anti-inflammatory and antioxidant activities that modulate aging [32].

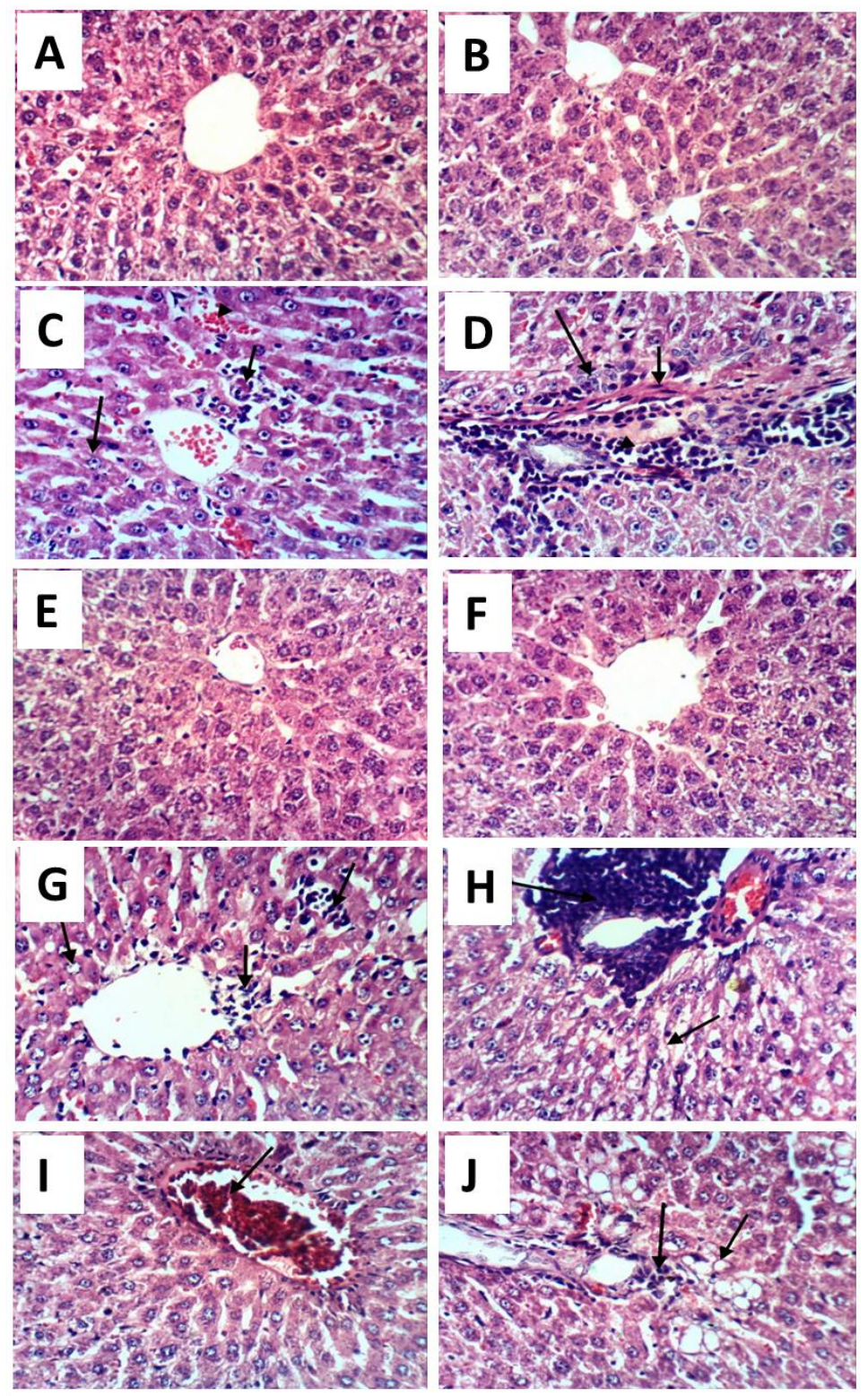

Figure 2. Photomicrographs of liver sections obtained from animals receiving different treatments. (A, B ) Normal histological structure showing central vein and concentrically arranged hepatocytes in normal control animals). C and D: liver section of D-galactose treated animal showing focal hepatic necrosis with inflammatory cell infiltration ( C, small arrow), vacuolated nuclei (C, large arrow), fibroplasia (D, small arrow), and oval cells proliferation (D, large arrow), E and F: liver sections from D-galactose treated animals that received 200 $\mathrm{mg} / \mathrm{kg}$ of macadamia nut extract showing the normal histological structure, $\mathrm{G}$ and $\mathrm{H}$ : liver sections from Dgalactose treated animals that received $200 \mathrm{mg} / \mathrm{kg}$ of macadamia leaves extract showing focal hepatic necrosis with inflammatory cell infiltration $(\mathrm{G})$, cytoplasmic vacuolation of hepatocytes (small arrow, $\mathrm{H}$ ) and portal infiltration with mononuclear cells (large arrow, H). I and J: liver sections from animals treated with Dgalactose and $200 \mathrm{mg} / \mathrm{kg}$ of macadamia shell extract showed some pathological changes such as congestion of the central vein (small arrow, I). 


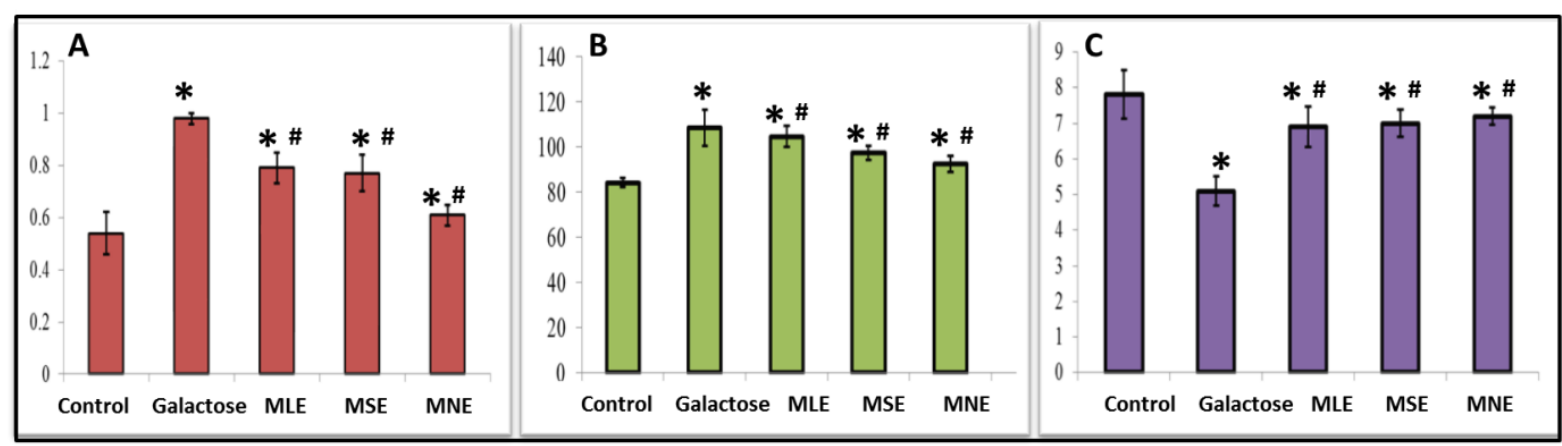

Figure 3. Effect of tested extracts on kidney function biomarkers in D-galactose accelerated aging animals. Kidney functions measured as serum concentration of A: creatinine $(\mathrm{mg} / \mathrm{dL}), \mathrm{B}$ : urea $(\mathrm{mg} / \mathrm{dL})$ and C: total serum protein $(\mathrm{g} / \mathrm{dL})$. Except for the control group, all animals were treated with D-galactose for 60 days 150 $\mathrm{mg} / \mathrm{kg}$ and received no further treatment (D-galactose ) or received $200 \mathrm{mg} / \mathrm{Kg} /$ day for 30 days of macadamia leaves extract (MLE), macadamia shells extract (MSE) and macadamia nut extracts (MNE).

* significantly different from control animals $(\mathrm{P}<0.05)$, \# significantly different from D-galactose treated animals $(\mathrm{P}<0.05)$.

Macadamia nuts and shells extracts also showed a high percentage of palmitoleic acid, which has been identified as a lipokine [33] with the ability to modulate different metabolic functions, including immune response and inflammations [34,35]. Additionally, the favorable modulation of serum lipid profile observed in human subjects consuming macadamia oil was associated with a high circulating level of palmitoleic acid [36]. Palmitoleic acid has also been described to reduce inflammation in different in vitro and in vivo studies [33,37]. However, it remains to be seen if the biological effects observed in this study can be attributed to the relatively high abundance of oleic and palmitoleic acid in MNE and MSE. Therefore, further investigation of the possible mechanism of the observed activity of macadamia extract and the specific role of oleic acid or palmitoleic acid in mediating these actions are strongly recommended.

\section{Conclusions}

This study indicates that macadamia nut may help preserve organ functions in integrity that deteriorate with aging and/or oxidative stress. This effect may be due to its high content of monounsaturated fatty acids (oleic and palmitoleic acids), which other investigators showed to have neuroprotective and anti-inflammatory properties. Additionally, our study indicates that waste products of macadamia (shells and leaves) may be good sources of the same bioactive metabolites, especially the shells which extract showed very promising results in reversing organ damage induced by $\mathrm{D}$-galactose in the model used.

\section{Funding}

This research received no external funding.

\section{Acknowledgments}

None.

\section{Conflicts of Interest}

The authors declare no conflict of interest. 


\section{References}

1. Zuza, E.J.; Maseyk, K.; Bhagwat, S.; Emmott, A.; Rawes, W.; Araya, Y.N. Review of Macadamia Production in Malawi: Focusing on What, Where, How Much Is Produced and Major Constraints. Agriculture 2021, 11, 152, https://doi.org/10.3390/agriculture11020152.

2. Mai, T.T.P.; Hardner, C.M.; Alam, M.M.; Henry, R.J.; Topp, B.L. Phenotypic Characterisation for Growth and Nut Characteristics Revealed the Extent of Genetic Diversity in Wild Macadamia Germplasm. Agriculture 2021, 11, 680, https://doi.org/10.3390/agriculture11070680.

3. Tu, X.; Wu, B.; Xie, Y.; Xu, S.; Wu, Z.; Lv, X.; Wei, F.; Du, L.; Chen, H. A comprehensive study of raw and roasted macadamia nuts: Lipid profile, physicochemical, nutritional, and sensory properties. Food Sci. Nutr. 2021, 9, 1688-1697, https://doi.org/10.1002/fsn3.2143.

4. Shuai, X.; Dai, T.; Chen, M.; Liang, R.; Du, L.; Chen, J.; Liu, C. Comparative Study of Chemical Compositions and Antioxidant Capacities of Oils Obtained from 15 Macadamia (Macadamia integrifolia) Cultivars in China. Foods 2021, 10, 1031, https://doi.org/10.3390/foods10051031.

5. Bolling, B.W.; Chen, C.-Y.O.; McKay, D.L.; Blumberg, J.B. Tree nut phytochemicals: composition, antioxidant capacity, bioactivity, impact factors. A systematic review of almonds, Brazils, cashews, hazelnuts, macadamias, pecans, pine nuts, pistachios and walnuts. Nutr. Res. Rev. 2011, 24, 244-275, https://doi.org/10.1017/S095442241100014X.

6. Bittner, K.; Rzeppa, S.; Humpf, H.-U. Distribution and Quantification of Flavan-3-ols and Procyanidins with Low Degree of Polymerization in Nuts, Cereals, and Legumes. J. Agric. Food Chem. 2013, 61, 9148-9154, https://doi.org/10.1021/jf4024728.

7. Castada, H.Z.; Liu, J.; Ann Barringer, S.; Huang, X. Cyanogenesis in Macadamia and Direct Analysis of Hydrogen Cyanide in Macadamia Flowers, Leaves, Husks, and Nuts Using Selected Ion Flow Tube-Mass Spectrometry. Foods 2020, 9, 174, https://doi.org/10.3390/foods9020174.

8. Insanu, M.; Hartati, R.; Bajri, F.; Fidrianny, I. Macadamia Genus: An Updated Review of Phytochemical Compounds and Pharmacological Activities. Biointerface Res. Appl. Chem. 2021, 11, 14480-14489, https://doi.org/10.33263/BRIAC116.1448014489.

9. Ain, H.B.U.; Saeed, F.; Barrow, C.J.; Dunshea, F.R.; Suleria, H.A.R. Food Processing Waste: A Potential Source for Bioactive Compounds. 2020; pp. 625-649, https://link.springer.com/content/pdf/10.1007/978-3030-30182-8_45.pdf.

10. Hanum, T.I.; Laila, L.; Sumaiyah, S.; Syahrina, E. Macadamia Nuts Oil in Nanocream and Conventional Cream as Skin Anti-Aging: A Comparative Study. Open Access Maced. J. Med. Sci. 2019, 7, 3917-3920, https://doi.org/10.3889/oamjms.2019.533.

11. Griel, A.E.; Cao, Y.; Bagshaw, D.D.; Cifelli, A.M.; Holub, B.; Kris-Etherton, P.M. A Macadamia Nut-Rich Diet Reduces Total and LDL-Cholesterol in Mildly Hypercholesterolemic Men and Women. J. Nutr. 2008, 138, 761-767, https://doi.org/10.1093/jn/138.4.761.

12. Garg, M.L.; Blake, R.J.; Wills, R.B.H.; Clayton, E.H. Macadamia Nut Consumption Modulates Favourably Risk Factors for Coronary Artery Disease in Hypercholesterolemic Subjects. Lipids 2007, 42, 583-587, https://doi.org/10.1007/s11745-007-3042-8.

13. Altamimi, M.; Zidan, S.; Badrasawi, M. Effect of Tree Nuts Consumption on Serum Lipid Profile in Hyperlipidemic Individuals: A Systematic Review. Nutr. Metab. Insights 2020, 13, https://doi.org/10.1177/1178638820926521.

14. Jones, J.; Rajaram, S.; Heskey, C.; Sirirat, R.; Clarke, A.; Oda, K.; Sabaté, J. Effect of Daily Macadamia Nut Consumption on Anthropometric Indices in Overweight and Obese Men and Women. Curr. Dev. Nutr. 2020, 4, 589-589, https://doi.org/10.1093/cdn/nzaa047_009.

15. Kumar, A.; Prakash, A.; Dogra, S. Protective effect of curcumin ( Curcuma longa ) against d -galactoseinduced senescence in mice. J. Asian Nat. Prod. Res. 2011, 13, 42-55, https://doi.org/10.1080/10286020.2010.544253.

16. Prajit, R.; Sritawan, N.; Suwannakot, K.; Naewla, S.; Aranarochana, A.; Sirichoat, A.; Pannangrong, W.; Wigmore, P.; Welbat, J.U. Chrysin Protects against Memory and Hippocampal Neurogenesis Depletion in D-Galactose-Induced Aging in Rats. Nutrients 2020, 12, 1100, https://doi.org/10.3390/nu12041100.

17. Azman, K.F.; Zakaria, R. d-Galactose-induced accelerated aging model: an overview. Biogerontology 2019, 20, 763-782, https://doi.org/10.1007/s10522-019-09837-y.

18. El Hawary, S.S.; Abubaker, M.; Abd El-Kader, E.M.; Mahrous, E.A. Phytochemical constituents and antityrosinase activity of Macadamia integrifolia leaves extract. Nat. Prod. Res. 2020, 1-6, https://doi.org/10.1080/14786419.2020.1849203.

19. Salimon, J.; Abdullah, B.M.; Salih, N. Hydrolysis optimization and characterization study of preparing fatty acids from Jatropha curcasseed oil. Chem. Cent. J. 2011, 5, 67, https://doi.org/10.1186/1752-153X-5-67.

20. Haas, M.J.; Bloomer, S.; Scott, K. Simple, high-efficiency synthesis of fatty acid methyl esters from soapstock. J. Am. Oil Chem. Soc. 2000, 77, 373-379, https://doi.org/10.1007/s11746-000-0061-1.

21. Pyka, A.; Sliwiok, J. Chromatographic separation of tocopherols. J. Chromatogr. A 2001, 935, 71-76 https://doi.org/10.1016/S0021-9673(01)00944-X. 
22. Rusu, M.E.; Georgiu, C.; Pop, A.; Mocan, A.; Kiss, B.; Vostinaru, O.; Fizesan, I.; Stefan, M.-G.; Gheldiu, A.-M.; Mates, L.; et al. Antioxidant Effects of Walnut (Juglans regia L.) Kernel and Walnut Septum Extract in a D-Galactose-Induced Aging Model and in Naturally Aged Rats. Antioxidants 2020, 9, 424, https://doi.org/10.3390/antiox9050424.

23. Nunez, J. Morris Water Maze Experiment. J. Vis. Exp. 2008, https://doi.org/10.3791/897.

24. Seidler, R.D.; Bernard, J.A.; Burutolu, T.B.; Fling, B.W.; Gordon, M.T.; Gwin, J.T.; Kwak, Y.; Lipps, D.B. Motor control and aging: Links to age-related brain structural, functional, and biochemical effects. Neurosci. Biobehav. Rev. 2010, 34, 721-733, https://doi.org/10.1016/j.neubiorev.2009.10.005.

25. El-Sabagh, O.A.; El-Toumy, S.A.; Mounir, R.; Farag, M.A.; Mahrous, E.A. Metabolite profiles of Pulicaria crispa and P. incisa in relation to their in-vitro/ in-vivo antioxidant activity and hepatoprotective effect: A comparative mass spectrometry-based metabolomics. J. Pharm. Biomed. Anal. 2021, 194, 113804, https://doi.org/10.1016/j.jpba.2020.113804.

26. Elbanna, A.; Nooh, M.; Mahrous, E.; Khaleel, A.; Elalfy, T. Extract of Bauhinia vahlii shows antihyperglycemic activity, reverses oxidative stress, and protects against liver damage in streptozotocininduced diabetic rats. Pharmacogn. Mag. 2017, 13, 607, https://doi.org/10.4103/pm.pm_4_17.

27. Abubaker, M.; Hawary, S.S. El; Mahrous, E.A.; El-Kader, E.M.A. Study of Nutritional Contents of Macadamia integrifolia Maiden and Betche Leaves, Kernel and Pericarp Cultivated in Egypt. Int. J. Pharmacogn. Phytochem. Res. 2018, 9, 1442-1445, https://doi.org/10.25258/phyto.v9i12.11189.

28. Chung, K.H.; Shin, K.O.; Hwang, H.J.; Choi, K.-S. Chemical composition of nuts and seeds sold in Korea. Nutr. Res. Pract. 2013, 7, 82, https://doi.org/10.4162/nrp.2013.7.2.82.

29. Nunes, E.; Rafacho, A. Implications of Palmitoleic Acid (Palmitoleate) On Glucose Homeostasis, Insulin Resistance and Diabetes. Curr. Drug Targets 2017, 18, 619-628, https://doi.org/10.2174/1389450117666151209120345.

30. Sadigh-Eteghad, S.; Majdi, A.; McCann, S.K.; Mahmoudi, J.; Vafaee, M.S.; Macleod, M.R. D-galactoseinduced brain ageing model: A systematic review and meta-analysis on cognitive outcomes and oxidative stress indices. PLoS One 2017, 12, e0184122, https://doi.org/10.1371/journal.pone.0184122.

31. Song, J.; Kim, Y.-S.; Lee, D.H.; Lee, S.H.; Park, H.J.; Lee, D.; Kim, H. Neuroprotective effects of oleic acid in rodent models of cerebral ischaemia. Sci. Rep. 2019, 9, 10732, https://doi.org/10.1038/s41598-019-47057z.

32. Serreli, G.; Deiana, M. Extra Virgin Olive Oil Polyphenols: Modulation of Cellular Pathways Related to Oxidant Species and Inflammation in Aging. Cells 2020, 9, 478, https://doi.org/10.3390/cells9020478.

33. Cao, H.; Gerhold, K.; Mayers, J.R.; Wiest, M.M.; Watkins, S.M.; Hotamisligil, G.S. Identification of a Lipokine, a Lipid Hormone Linking Adipose Tissue to Systemic Metabolism. Cell 2008, 134, 933-944, https://doi.org/10.1016/j.cell.2008.07.048.

34. Guillocheau, E.; Legrand, P.; Rioux, V. Trans-palmitoleic acid (trans-9-C16:1, or trans-C16:1 n-7): Nutritional impacts, metabolism, origin, compositional data, analytical methods and chemical synthesis. A review. Biochimie 2020, 169, 144-160, https://doi.org/10.1016/j.biochi.2019.12.004.

35. de Souza, C.O.; Vannice, G.K.; Rosa Neto, J.C.; Calder, P.C. Is Palmitoleic Acid a Plausible Nonpharmacological Strategy to Prevent or Control Chronic Metabolic and Inflammatory Disorders? Mol. Nutr. Food Res. 2018, 62, 1700504, https://doi.org/10.1002/mnfr.201700504.

36. Frigolet, M.E.; Gutiérrez-Aguilar, R. The Role of the Novel Lipokine Palmitoleic Acid in Health and Disease. Adv. Nutr. An Int. Rev. J. 2017, 8, 173S-181S, https://doi.org/10.3945/an.115.011130.

37. Souza, C.O.; Teixeira, A.A.S.; Biondo, L.A.; Silveira, L.S.; Calder, P.C.; Rosa Neto, J.C. Palmitoleic acid reduces the inflammation in LPS-stimulated macrophages by inhibition of NFKB, independently of PPARs. Clin. Exp. Pharmacol. Physiol. 2017, https://doi.org/10.1111/1440-1681.12736. 